\title{
Incommunicabilité conjugale et violence feutrée contre la femme dans le roman : le bonheur conjugal de Taher Ben Jelloun.
}

\author{
Soliman Magdy ( Amany) \\ Professeur adjoint au Département de français \\ faculté des langues AL ALSUN, Université de MINIA
}

\section{Introduction}

Taher Ben Jelloun a publié en 2012 un roman intitulé "Le bonheur conjugal". Ce roman a laissé couler beaucoup d'encre en France et dans les milieux littéraires du monde entier. L'histoire du roman relate la vie infernale qui unit un peintre marocain d'origine bourgeoise à une jeune femme issue du milieu de berbère immigrés. La valeur connotative euphorique du titre suscite l'intérêt du grand public, ce qui nous a encouragée par la suite à entamer une recherche sur l'incompréhension conjugale. Il nous a semblé utile d'aborder un tel sujet critique surtout à un certain moment de l'histoire de l'humanité où l'on enregistre des cas importants d'incommunicabilité entre les époux notamment que les problèmes conjugaux ne cessent de s'accentuer. Jelloun pose ici avec insistance cette question: pourquoi la femme demeure t'elle la part la plus faible dans la société? Pourquoi demeure $t^{\prime}$-elle la cible de la maltraitance malgré la progression des sociétés vers plus de modernité? Ou se trouve la lacune? Pourquoi elle et non pas l'homme qu'on considère injustement comme la cause du malheur lorsque la vie conjugale échoue?

Nous nous sommes penchée sur cette problématique pour chercher une réponse valable et acceptable à ces questions qui nous irritent.

Notre problématique sera discutée selon trois axes principaux. L'approche psychosociale s'appuie sur les travaux de Michael Kaufman qui nous invite à comprendre les raisons du comportement agressif dans les sociétés patriarcales et qui encouragent les hommes 
à user de la violence contre la femme. l'approche psychanalytique se base sur les travaux de dr. Jean Cautaerts, et ceux de MarieFrance Hirigoyen. Les deux psychanalystes ouvrent la porte de la science pour initier le lecteur aux différents types de harcèlement dont la femme peut être victime soit dans le couple soit en société. En ce qui concerne, l'approche neurologique, l'investigation sera basée sur les travaux de Dr. Louann Brizendine. La neurologie est une approche nouvelle qui se base sur l'étude du cerveau humain au moyen de l'imagerie cérébrale pour donner des explications au comportement humain. C'est une approche interdisciplinaire entre la biologie, la neurologie et l'ethologie. Ses résultats de recherche sont ultra-fascinants pour l'explication des comportements des personnes en société. Ils seront d'une aide inestimable pour la compréhension des attitudes des époux dans le couple, objet de notre recherche.

Notre plan de travail se divise en quatre points. Le premier point présente au lecteur les étapes normales de la constitution d'un couple ordinaire depuis le coup de foudre jusqu'à la résurgence des conflits mortels. Le second point est une tentative de compréhension et d'explication de la problématique sous une approche neurologique. Les travaux de Dr. Louann Brizendine nous ont été d'une préciosité inestimable parce qu'ils nous ont permis de comprendre les différences anatomiques et neurobiologiques entre les deux cerveaux masculins et féminins. La violence feutrée contre la femme, son mutisme contre le harcèlement moral dont elle est victime seront étudiés en détail. Nous allons découvrir les raisons neurologiques qui sont à l'origine de l'agressivité masculine envers la femme. Le troisième pont mettra l'accent sur les raisons qui maintiennent la violence sociale envers la femme. Le quatrième point étudiera les carences affectives des deux époux. L'analyse psychanalytique nous fera découvrir la théorie du harcèlement moral, psychique et sexuel, un engrenage de maladies psychiques qui passe de la société vers le couple.

Jelloun a voulu présenter les deux points de vue des deux époux à travers une narration à deux volets. La narration fut assumée par un narrateur auto-diegetique dont l'instance narrative est la troisième personne du singulier : "Le peintre", "il", "la femme du peintre", "foulane" ce qui reflète peut être la distance psychique qui sépare le 
peintre de sa femme. Celui-ci a assumé la narration de la première partie du roman où il relate l'histoire de son échec conjugal selon son propre point de vue: un homme d'origine arabe, bourgeois, de culture européenne, amoureux d'une jeune femme de même origine mais de caste et de culture totalement contradictoire. Apparemment, cette différence de caste et de culture a joué un rôle important dans la construction de l'incommunicabilite. Mais nous avons poussé la recherche un peu plus profondément pour pouvoir découvrir les raisons neurobiologiques qui maintiennent constamment l'incompréhension entre l'homme et la femme partout dans le monde.

La seconde partie de la narration fut assumée par l'épouse du peintre qui a voulu se défendre acharnement contre les accusations de son mari.

Les deux narrateurs ont préféré gardé l'anonymat. Cette distanciation de la désignation traduit peut-être le refus de chacun pour l'autre ou la distance morale qui sépare dorénavant les deux partenaires, jadis amoureux l'un de l'autre, ennemis après deux ans de mariage. Le peintre se désigne par "il" et sa femme le désigne par Foulane, la transcription écrite du mot arabe : une personne inconnue. Ce choix de part et d'autre ne peut expliquer que le refus psychique mutuel que nourrissent les deux époux. Inconsciemment, chacun refuse l'existence de l'autre dans sa vie ou bien refuse le lien conjugal qui les lie. Chacun nourrit peut-être l'espoir de détruire l'autre ou au moins de se défaire de ce lien qui les étouffe. La distanciation traduit son besoin de fuir ou plutôt de vouloir évaluer posément sa vie précédente pour mieux analyser les raisons de cet échec.

La configuration de la femme dans l'œuvre de Ben Jelloun est passée par deux étapes principales. Au début de son œuvre, Jelloun avait présenté la femme sous une configuration mutilée. A l'image de la femme marocaine dans la société postcoloniale, Jelloun présente une femme mutilée, piétinée, blessée dans son amour propre et dénouée de tous ses droits les plus élémentaires. Jelloun présente une image démolie, piétinée, rabaissée par rapport à l'homme qui maintient son destin et abuse d'elle à son gré. Il est possible que l'écrivain marocain aurait voulu mettre l'accent sur cette situation humiliante de la femme pour en faire le porte-parole ou le défenseur 
dans une société patriarcale par excellence. Cette société n'avait même pas la conscience de piétiner la femme. Celle-ci n'était qu'un objet à acquérir comme l'héritage. Le détenant du pouvoir abusait d'elle comme il lui plaît. Jelloun a voulu être la voix qui retentit dans la conscience arabe pour affranchir la femme de la sujétion.

La seconde phase de cette configuration correspond aux évolutions culturelles et instructives qui ont eu lieu au sein de la société marocaine après la colonisation. À l'encontre de la femme démolie, il présente cette fois-ci une personne sûre d'elle-même, capable de confronter et même de braver les circonstances difficiles qui lui sont imposées. En montrant une femme combattant le harcèlement moral de la société masculine, Jelloun nous présente une nouvelle version d'une femme qui a arraché sa liberté du système patriarcal accablant.

Malgré les tentatives interminables d'entente conjugale, la problématique de l'incommunicabilite entre les époux demeure au cœur du problème. Taher Ben Jelloun nous donne ici l'exemple d'un couple qui a été fondé sur un espoir grandissant d'amour et de prospérité. Après le coup de foudre de la rencontre, s'installe l'amour aveugle et les promesses évanescente de bonheur. La lune de miel résiste deux ans aux secousses des problèmes. L'amour craque après seulement deux années en s'achoppant aux labyrinthes d'une vie conjugale semée d'embûches.

Nous allons nous pencher sur les raisons de cette incommunicabilité pour trouver où se trouve la lacune qui mènera par la suite à l'installation de cette incompréhension, puis à la naissance de la violence; une violence feutrée au début, verbale, ensuite mais qui ne tardera pas à tourner en violence psychique. Nous verrons comment les plus simples malentendus quotidiens et passagers peuvent s'accumuler pour, enfin de compte, construire un mur entre les deux partenaires. Nous verrons comment l'amour se désintègre et explose à cause de l'envahissement des malentendus.

\section{Premier point:}




\section{ÉTAPES DE LA CONSTITUTION DU COUPLE:}

\section{1) PREMIÈRE PHASE DU MARIAGE: a) Fusion de l'amour:}

Le héros de l'histoire est un peintre de renommée mondiale, après avoir mené une vie mondaine et libérale autour du monde, décide a l'âge de trente-huit ans de fonder une famille. Il tombe amoureux d'une jeune fille de vingt-quatre ans, plus jeune que lui de quatorze ans:

"Il considérait qu'à trente-huit ans, épouser une jeune fille de vingt-quatre ans était quelque chose d'exceptionnel??un??privilège??réservé??à??peu??d'élus" Jelloun, le bonheur conjugal, Paris, Gallimard, 2012, p. 7)

Après le coup de foudre, la lune de miel, les promesses flatteuses, le couple jouit d'un bonheur parfait: "Ils avaient tort de médire, pendant deux longues et douces années, le peintre et sa femme furent le couplé le plus heureux du monde" ( jelloun, 2012,p. 9)

La jeune dame était intensément amoureuse et décidée à faire réussir cette vie conjugale: " elle savait le rendre bon, avait appris très vite comment s'adapter à ses manies, ses habitudes, ses lubies. Elle les acceptait avec sourire et parfois en se moquant gentiment. Jamais l'ombre d'une contrariété. Météo Parfaite! Disait elle en souriant"( jelloun, 2012,p. 17)

Malgré la patience de la jeune mariée et son insistance à faire réussir son projet de mariage, les malentendus n'ont pas tardé à surgir un jour après l'autre à cause de la grande différence sociale qui sépare leurs deux mondes respectives. Le peintre était né à Fes, une ville marocaine bourgeoise. Il était descendant d'une grande famille bourgeoise dont le grand père maternel était professeur à l'université de Quayrawaan:" Les gens de Fes se croient supérieurs à tous les autres Marocains. Ils regardent le reste du Maroc de haut, comme si leurs traditions devaient être adoptés par tout le monde, comme si tout le Maroc devait cuisiner comme eux, s'habiller comme eux, parler comme eux. Leur intolérance est naturelle, leur mépris 
affiché, ils ne sont pas méchants, juste cynique"( Jelloun, 2012,p. 293)

Le père du peintre était farouchement opposé à ce mariage, il jugeait que cette alliance était incompatible. Sa mère était contrariée par ce choc des classes, elle ne comprenait pas " comment une dame de la haute bourgeoisie de Fès comme elle, pouvait-elle accepter de se retrouver en compagnie de campagnards qui ne parlaient même pas bien l'arabe?" (Jelloun, 2012,p. 58)

Également, les autres membres de la famille étaient choqués par cette alliance. Ils étaient beaucoup plus acerbes dans la manifestation de leurs refus:" les autres membres de la famille partageaient la même position, il pouvait le lire sur??les??visages??crispés??des??uns??et??des??autres" Jelloun, 2012,p. 10). Même les invités pensaient qu'il fallait être fou" pour vouloir réunir deux mondes aussi différents. " ( Jelloun, 2012,p. 5)

Cette disjonction totale entre leurs deux mondes a engendré, par conséquent, une hostilité ostentatoire entre les deux familles dès le début du mariage. Le jeune couple avait nourri l'espoir de sauter sur les barrages sociaux qui les séparent mais comme une conséquence normale à cette scission de classe, les deux époux n'avaient jamais réussi à former un cercle d'amis commun:

" nous n'avions pas les mêmes amis, non seulement pour des raisons de générations, mais aussi de classes sociales. Les miens sont presque tous issus du milieu immigré. Les siens sont des intellectuels, des artistes internationaux, des écrivains, des politiques, tous très imbus d'eux. Ils regardent soit avec condescendance, soit avec cette gentillesse avec laquelle on traite les enfants qui se mêlent aux grandes personnes". (Jelloun, 2012, p. 333)

Devant le monde bourgeois auquel il appartient, le peintre était désolé de ne pas pouvoir surmonter ce barrage social. Devant les Fassis qui se croient supérieurs et qui n'ont aucune considération pour les autres, il était désolé, " désolé que sa famille soit raciste, désolé que ma famille soit si peu éduquée, désolé que j'appartienne à 
cette tribu où l'on n'avait pas appris les bonnes manières de Fes."( Jelloun, 2012, p. 334)

\section{b) Résurgence des malentendus: Différence de culture:}

Les scènes explosives n'ont pas retardé à surgir. Le peintre reçoit en cadeau une très belle nappe de Fès brodée à la main qui avait requis à ses yeux une valeur inestimable: " cette pièce avait maintenant à ses yeux une valeur inestimable: non seulement elle était belle et unique, mais elle faisait partie du patrimoine familial." ( Jelloun, 2012, p. 53)

Cette belle nappe avait été vite jeté dans le panier à linge, soit par indifférence, soit par ignorance. La jeune dame n'a pas su apprécier la valeur exacte de la pièce artistique. Quand son mari essaye de lui faire comprendre sa vraie valeur, elle lui répond: " c'est quoi cette vieillerie, ma maison n'est pas un bazar." ( Jelloun, 2012, p. 53)

Le mari n'avait jamais aimé par exemple les musiques folkloriques pleine de stridence et de disharmonie, pourtant, c'était le genre préféré chez sa femme.

C'est ainsi que les malentendus vont en recrudescence surtout lorsque ce mari, lassé des disputes, décide de fuir la maison. Estimant qu'il incombe uniquement à la femme la responsabilité du ménage, il prend le large, fuyant ainsi ses responsabilités de père et d'époux.

Sa femme commence à se plaindre. La vision du couple commence à s'avérer contradictoire quand le père de la famille se soustrait à ses responsabilités sous prétexte que les disputes quotidiens ne lui fournissent pas la paix:

" il n'est tout simplement jamais Là! Auprès des enfants, je suis obligée d'assumer les deux rôles, le père et la mère. (.....) il prétexte qu'il doit travailler à son atelier, qu'il a des expositions, et on ne le voit jamais. Quand par hasard, il revient, il est toujours de mauvaise humeur, il crié, hurle et frappe les enfants" ( Jelloun, 2012, p. 54) 
Même lorsque le mari prend l'initiative de consulter un psychiatre pour déterminer les lacunes de leur vie conjugale et y remédier, son épouse a catégoriquement refusé cette démarche. En se montrant réticente, elle a perdu une chance en plus de sauver son foyer de la destruction. La différence de culture a gagné une fois de plus parce qu'elle a bloqué une bonne solution qui aurait pu sauver le foyer ou au moins, désamorcer les conflits:

" il sourit et expliqua (au psychiatre) que la culture de sa femme ne la prédisposait pas à une telle démarche. Au mieux, elle consulterai des charlatans qui lui donnerait des trucs à faire, du genre de l'encens à brûler(.....), des talismans. Ces pratiques magiques, fréquentes chez des gens analphabètes et attachés à leur tradition ancestrale dans les régions de montagnes, confortaient son irrationalité." ( Jelloun, 2012, p. 128)

Cette différence de culture, ajoutée à une très grande différence d'âge ne pouvait engendrer que des malentendus en spirale. Cette dame qui résiste à la compréhension de la mentalité de son mari s'est trouvée en réalité dans une situation complexe d'infériorité face au monde trop plein de certitude de celui- ci.

Dans son œuvre intitulé: " De la dépendance amoureuse à la liberté d'aimer" , Hélène Roubeix nous explique que toute relation conjugale passe par quatre phases principales. Selon elle, la première phase de l'amour se caractérise par la fusion et un trop plein de dépendance. Ces débuts fusionnels impliquent une soumission d'un partenaire à la volonté de l'autre pour que l'entente soit parfaite. Pourtant, cette soumission s'avère dangereuse parce qu'elle se transforme en prison entravant le processus d'évolution du couple vers les phases suivantes:

" la dépendance amoureuse est basée sur l'utilisation inconsciente de l'autre comme d'un objet pour combler ses propres manques et répondre à ses propres attentes." ( roubeix, de la dépendance amoureuse à la liberté d'aimer, Paris, Saint Germain, eyrolles, 2008, p. 17)

Après le passage de la première phase caractérisée par la fusion et la soumission d'un des partenaires à l'autre, le couple, objet de notre 
étude, passe à la deuxième phase, celle de la "contre dépendance". Le couple demeure prisonnier dans les barbelés de cette phase jusqu'à la fin de sa vie conjugale relatée dans l'histoire de Ben Jelloun. Cette phase se caractérise par l'abondance des conflits. Lorsque l'épouse du peintre refuse les barbelés de la dépendance, et refuse de vivre en fonction des exigences de son mari, la phase des conflits prend la relève:

"En fait, il s'arrangeait pour que je sois dépendante de lui, de sa générosité, comme si j'étais une étrangère ou un de ses enfants" ( Jelloun, 2012, p. 312)

\section{2) Deuxième phase du mariage:}

\section{phase des conflits ou la contre dépendance ${ }^{1}$ manque de respect:}

Le mari commence à lui manquer de respect, même en public. Quand elle essaie de prendre part aux conversations, il avait l'audace de la faire taire même devant les invités: "Mon mari avait pris l'habitude de me faire taire quand je parlais à table avec des invités. " ( Jelloun, 2012, p. 57)

Ce genre de harcèlement moral demeure en recrudescence d'une manière parfois explicite, en public, et beaucoup d'autre fois de façon implicite: " j'étais sa muse, son esclave, sa chose, sa jolie petite chose qu'il exhibait dans les réceptions. "( Jelloun, 2012, p. 303)

Malheureusement, le peintre profitait de sa femme comme si elle était une Chose, ou un Objet, non pas une personne en chair et en os. Après le manque d'attention, le manque de respect, le manque de responsabilité, il prend le large. L'infidélité fraye son chemin vers le couple. Il cherche son repos dans des relations extra conjugales:

" Il reprit très vite ses habitudes de célibataire sans se soucier de ce que je pouvais ressentir. (....) Il rentrait tard, et je sentais sur lui le parfum d'autres femmes. Je ne disais rien, j'avalais des couleuvres. Je regardais mes enfants, et je pleurais en silence". (Jelloun, 2012, p. 288) 
Dans sa version à lui, le peintre ne nie pas son infidélité. Il relate avec précision ses diverses relations extraconjugales comme s'il s'en vantait. Il se souvient avec beaucoup de délectation de ses nuits d'amour et du nombre incalculable de ses maîtresses:" Il pensa au cortège de celles qui l'avaient aimé et qu'il avait aimées" ( Jelloun, 2012, p. 58)

Au lieu de parler pour communiquer les raisons des dissonances et trouver des solutions raisonnables pour maintenir l'équilibre du couple, monsieur le mari préfère fuir. Au lieu de confronter courageusement ses responsabilités de père de famille et d'époux il tourne le dos et fuit: " pour éviter les disputes, il commença à la fuir et à nouer clandestinement des liens avec des femmes aimantes, des femmes qui l'admiraient en tant qu' artiste et en tant qu'homme. "( Jelloun, 2012, p. 86)

Ce que les hommes ne comprennent pas, c'est qu'ils compliquent de la sorte leurs problèmes en les multipliant. La femme ne peut pas pardonner l'infidélité. Celle-ci ne fait que creuser de plus en plus le fossé entre eux et d'élever le mur de l'incommunicabilité. Il ne peut pas mesurer l'ampleur de l'attaque morale qu'il assène à sa femme quand il la trahie: " se sentant trahie, elle cherchait par tous les moyens à se venger, à lui rendre au quintuple le mal qu'il lui faisait. Il restait muet, fuyait et la laissait dans son désarroi." ( Jelloun, 2012, p. 163)

Jusqu'ici, la parole a perdu sa fonction normale de communication saine, elle acquiert le stade de violence verbale entre les époux: " malheureusement, beaucoup de limites ont été dépassées des deux côtés. Il n'y a plus de respect entre nous." ( Jelloun, 2012, p. 163)

Le fossé s'élargit de plus en plus. Il s'est rempli de malentendus, de différence de goût, de manque de respect et aussi de manque de responsabilité. Il fut comblé en fin de compte par l'épée de l'infidélité. Toute cette trajectoire était semée de violence. Au début, une violence muette, implicite, insinuee par des gestes, des comportements, de désapprobation avant de devenir affichée et sans vergogne. 
Le peintre, en tant que Homme, et par dessus tout Arabe, ne se sentait pas coupable. En toute simplicité, il jette la responsabilité des dissonances sur sa femme. Son orgueil masculin ne lui permet pas de se sentir ni fautif ni responsable de la décadence du couple. Sa dureté émotionnelle le rendait incapable de se sentir en mesure de partager cette responsabilité: "A aucun moment, le peintre ne se sentait coupable. Il ne faisait rien de mal, cherchait son équilibre en dehors de son couple qui fonctionnait par intermittence. " ( Jelloun, 2012, p. 165)

Il paraît clair maintenant qu'aucun des deux partenaires n'a pu évoluer vers le stade rationnel de "l'interdépendance"2. Leur incapacité réciproque à surmonter leur conflit était dû à une incapacité à communiquer les raisons de leur gêné. La mort de la parole laisse la place à l'installation de l'incommunicabilité.

Ben Jelloun relève ici plusieurs questions importantes: " pourquoi les couples s'appauvrissent à ce canal de communication pour résoudre leurs malentendus? Pourquoi l'accusation de la femme est la solution la plus simple? Pourquoi la société endosse à la femme l'habit de la fouteur de trouble? Pourquoi la société responsabilise uniquement la femme lorsque le couple craque?

Nous avons essayé de trouver une réponse valable à ce dilemme. Nous nous sommes appuyée sur les études de neurobiologie menées par Dr. Louann Brizendine pour pouvoir comprendre comment fonctionne le cerveau masculin et quelle différence existe t'-elle entre les deux cerveaux: féminin et masculin pour trouver une réponse aux questions soulevées par l'écrivain:

" pourquoi ne sommes-nous jamais parvenus à nous parler, à discuter sans nous disputer, à dialoguer sans vouloir tout casser, bref, à négocier et à vivre intelligemment ensemble? " ( jelloun, 2012, p. 142)

\section{II) DEUXIÈME point:}

Différences neurobiologiques entre le cerveau féminin et masculin: 
La fondatrice de la woman's mood and hormone clinic, premier centre pour le soin du cerveau et l'étude de l'action des hormones, Dr. Louann Brizendine nous explique les raisons fondamentales de cette différence mentale qui perpétuent les dissonances entre les deux sexes. Dans son ouvre " les secrets du cerveau féminin"3, elle montre que le cerveau est d'abord unisexe du côté biologique, et neuro anatomique:

" ( A la naissance) le code génétique des hommes et celui des femmes sont semblables à 99\%. Sur les quelques trente mille gènes que compte le génome humain, une différence de $1 \%$ entre les sexes est minime". (brizendine, 2008, p. 9)

Le résultat de cette recherche contrecarre les dires sociales que la femme est moins intelligente que l'homme, par conséquent, celui-ci doit lui rester supérieur et la dominer en permanence.

\section{a) Disposition naturelle à la communication du cerveau féminin:}

La spécialiste en neurobiologie nous explique pour quelles raisons les femmes sont-elles capables de mieux s'exprimer par le langage pour communiquer ce qu'elles ressentent:

" Dans le centre du langage et celui de l'audition, (...), les femmes ont $11 \%$ de neurones en plus. L'hippocampe, principale plateforme des émotions et de la formation de la mémoire, est aussi plus volumineux dans le cerveau féminin, tout comme les réseaux de circuits du langage et de l'observation des émotions chez autrui." ( brizendine, 2008, p. 10)

Cela veut dire que la femme possède un cerveau mieux branché sur la communication verbale. La femme met au premier plan les valeurs de la communication, de la sensibilité émotionnelle et de l'attention aux autres. Elle est mieux branchée socialement. Ses circuits cérébraux sont ainsi façonnés:

" le cerveau féminin et le cerveau masculin ne sont pas programmés de la même manière pour??l'empathie2017 ( brizendine, 2008, p. 43) 
Selon Dr. Brizendine, le cerveau féminin possède de formidables aptitudes verbales: " la capacité de s'impliquer à fond dans l'amitié, un don pratiquement demiurgique pour déchiffrer les émotions et les états d'âme d'après l'expression du visage et le son de la voix, la capacité de désamorcer les conflits. Tout cela est programmé dans le cerveau féminin. Ce sont des dons innés que beaucoup d'hommes, (...) ne possèdent pas2017 ( brizendine, 2008, p. 21)

\section{b) Armature mentale rigide du cerveau masculin}

Dr. Brizendine nous explique également que l'armature mentale de l'homme est beaucoup plus rigide que celle de la femme. De par la construction de ses neurones, l'homme est incapable d'être emphatique. Il est peu enclin aux échanges verbaux et sentimentaux. Son manque de fluidité verbale associée à une armature psychique rigide semble être une des causes essentielles de la non compréhension entre les deux sexes.

Michael Kauffman, dans son œuvre intitulé "the construction of the Masculinity"4 approuve les résultats de la recherche de Dr. Brizendine. Il assure que l'armature mentale de l'homme est basée en principe sur le manque d'empathie. Les hommes sont incapables de ressentir ce que les autres éprouvent. Ils sont incapables de percevoir les besoins des autres. Si les hommes sont égoïstes, peu enclin à l'affection, rien donc ne les empêche d'user de la violence envers autrui. S'ils sont incapables de ressentir le mal qu'ils infligent aux autres, comment peuvent-ils percevoir que l'infidélité est une violence psychique muette contre la partenaire. Il ne réalise pas que ce comportement violent dont ils usent porte du mal à autrui. Leur armature mentale est construite de rigidité. Lorsqu'un homme frappe sa femme ou se remarie, il se cherche bien sûr des excuses, " mais une partie du problème provient ici du fait qu'il ne ressent peutêtre vraiment pas la douleur qu'il cause." ( kauffman, 1985, p. 280)

L'homme a une moindre capacité à lire et à comprendre les sentiments d'autrui. Jelloun avait déjà donné des exemples multiples dans son roman sur cette pauvreté sentimentale entre les époux: 
" il était incapable du moindre compliment, pas un mot gentil le matin, pas un geste de tendresse au coucher, rien, il vivait dans son monde et je devais exister dans son ombre, me faire toute petite dans son ombre" ( Jelloun, 2012, p. 281)

Les hommes ignorent qu'un petit mot tendre est capable de changer complètement l'humeur de son épouse et par suite changer le cours de leur vie. Le peintre ne savait malheureusement pas dire un mot doux:

"Dire un mot doux devait lui cô̂ter. Alors, il s'absentait, se fermait sur lui-même. "( Jelloun, 2012, p. 281)

L'explication scientifique et neurobiologique de Brizendine ne veut nullement dire que nous trouvons des excuses quelconque au manque de sentimentalité d'un époux envers son épouse. Nous essayons uniquement de comprendre pourquoi l'incompréhension dégénère petit à petit en violence. Il paraît qu'ils sont innés incapable de communiquer. Depuis la phase intra utérine le cerveau masculin reçoit une dose importante de testostérone qui le transformera d'un cerveau unisexe à un cerveau typiquement masculin, comme l'explique Dr. Brizendine:

" un afflux important de testostérone va faire de ce cerveau unisexe un cerveau masculin, en aneantissant certaines cellules des centres de la communication et en augmentant le nombre des cellules des centres de la sexualité et de l'agressivité. " ( brizendine, 2008, p. 36)

Quand les garçons se servent du langage, ce n'est pas pour transmettre ou recevoir des paroles au sens ordinaire. Il n'utilise pas la langue comme vecteur de communication, au contraire, quand ils s'en servent, ils le font "pour donner des ordres, obtenir ce qu'ils veulent, se vanter, menacer, ignorer la suggestion d'un camarade et empêcher l'autre de parler." ( brizendine, 2008, p. 82)

Leur cerveau, imprégné de testostérone ne recherche pas autant la communication sociale. Le monde masculin n'est en rien gêné par les conflits qui peuvent éclater en société entre eux: 
"la perspective d'un conflit ne les gêné pas et la constitution de leur personnalité passe par la compétition." ( brizendine, 2008, p. 50)

En société, les femmes ignorent donc que la recherche d'intimité et de communication n'est propre qu'au sexe féminin. Cherchant en vain les touches douces et sentimentales de leurs maris, elles s'achoppent à l'armature mentale rigide des hommes et leurs incapacités à exprimer leurs sentiments. Alors, la déception s'installe et la femme croit que son mari ne l'aime plus et ne cherche plus sa compagnie, ce qui la blesse profondément. Elle commence à le lui reprocher. De sa part, le mari, étant incapable par sa nature cérébrale à la combler, ou bien, il l'attaque, ou bien, il la fuit. Il l'attaque pour qu'elle le laisse en paix, sinon, il s'enfuit pour garder son équilibre psychique. Dr. Brizendine a cité que la réaction masculine face à une menace ou à un stress est caractérisée par, soit la lutte, soit la fuite:

" la personne soumise à un stress ou à une menace va attaquer la source de cette menace (son épouse par exemple), si elle a quelque chance de gagner, sinon, elle va fuir la situation." ( brizendine, 2008, p. 83)

Donc, face au conflit, les hommes ne dialoguent pas, ne se donne même pas l'occasion de comprendre pourquoi le malentendu a eu lieu. Ils ne sont même pas disposé à vivre ce genre d'explication de peur d'être atteint dans leur orgueil ou par refus interne de reconnaître leurs fautes. En bloquant les moyens d'expression, ils ne font que compliquer les situations de crise en croyant faussement que cette fuite va remédier aux dissonances. Ils ne cherchent pas de solutions, fuient, s'isolent, laissant l'épouse dans le labyrinthe du doute et du stress.

Fuir la maison et le contact était l'option du peintre, héros de l'histoire de Ben Jelloun. Il s'isole dans son atelier sous prétexte de débordement de travail et de commandes accrues de toiles. Cette fuite ne fait que compliquer la situation parce que celui-ci a pris cette décision abrupte, non pas pour réfléchir à tête reposée mais pour vite nouer des relations faciles avec des étudiantes étrangères, toutes 
émues par ses avances discrètes: "(les étudiantes) étaient toutes sensibles à sa disponibilité et cédaient facilement à ses avances discrètes." ( Jelloun, 2012, p. 155)

Le romancier a voulu mettre en exergue les relations extra conjugales du peintre en leur consacrant tout le chapitre quinze du roman, qui va de la page cent quarante-cinq jusqu'à la page cent soixante-six: "Il ne se souvenait plus des noms mais de leurs parfums, de leurs gestes intimes. "( Jelloun, 2012, p. 155)

Malgré ce désordre de relations sexuelles extra conjugale, le peintre n'avait aucun sentiment ni de responsabilité ni de remords:

" ils savaient que cela ne pouvait pas durer, que la réalité allait les rattraper, surtout, lui, qui trahissait sa femme et ne se sentait pas coupable." ( Jelloun, 2012, p. 198)

\section{c) violence feutrée contre la femme, une violence muette:}

Personne ne peut mesurer l'ampleur de l'attaque morale subie par une femme qui est sûre de l'infidélité de son mari. La profondeur de la blessure est incommensurable. Ce fut un des genres multiples de mort psychique, de violence indirecte et passive contre la femme. Le mari qui délaisse sa femme, la trahit, lui inflige une des violences passives les plus atroces. Il l'exécute sans procès. Cette sciure psychique est due au blocage de l'afflux de l'hormone " ocytocine". L'absence du mari prive en réalité la femme de l'afflux de cette hormone responsable du bien-être dans le cerveau féminin. La privation de l'écoulement de cette hormone dans les circuits cérébraux de la femme gêné le sentiment de repos chez celle- ci:

" le cerveau est quasiment en état de manque."( brizendine, 2008, p. 136)

Les zones cérébrales de la femme demeurent désespérément en quête de l'être aimé :

"le manque s'installe, comme dans un sevrage de la drogue." ( brizendine, 2008, p. 136) 
Les centres d'intérêt de la femme, ses convictions, ses loisirs, et ses traits de caractère sont maintenus en état de manque affectif et psychique. Elle se sent menacée dans son bien-être.

La douleur psychique de cet abandon équivaut, selon la neurobiologie, à la douleur physique car il active les mêmes circuits cérébraux dans le cerveau:

"l'abandon équivaut en réalité à une douleur physique, car il active les mêmes circuits cérébraux. L'image du cerveau de personnes que le partenaire venait de quitter révèle que l'ébullition chimique de l'amour à laisser la place à la morne biochimie du deuil et du chagrin. Sans les afflux de dopamine, le cerveau est obscurci par le nuage noir de réponse dépression-désespoir. " ( brizendine, 2008, p. 137)

Cet abandon suscite chez la femme abandonnée un état d'angoisse et de stress. Les zones cérébrales de la femme restent désespérément en quête du mari perdu. Le taux de l'hormone du stress augmente considérablement dans le cerveau féminin:

"Quand une relation est menacée ou détruite, le niveau de certaines substances neurochimiques du cerveau féminin, comme la sérotonine, la dopamine, et l'ocytocine (hormone de l'attachement) baisse considérablement; et le cortisol, l'hormone du stress, prend le relais. La femme commence à se sentir inquiété. Elle redoute confusément d'être rejetée et abandonnée. (...) Les modifications hormonales la poussent alors à s'imaginer, terrifiée, que la relation est finie." ( brizendine, 2008, p. 136)

Dr. Brizendine avance que les femmes ignorent que cette recherche d'intimité et de communication est propre au sexe féminin. Celles-ci cherchent constamment un mirage que les hommes ne possèdent pas.

Actuellement, nous sommes au cœur du dilemme. La problématique demeure sans réponse. Pourquoi la femme demeure telle la source du malheur si la science prouve qu'elle est victime? 
Si la science moderne explique que l'escalade de la tension provient de la nature mentale rigide de l'homme, pourquoi cette tension mène $\mathrm{t}$ - elle à la recrudescence de la violence contre la femme dans la société en général et contre l'épouse en particulier en sein du couple. Nous nous expliquons. Si, en cas de conflit ordinaire, le mari se révèle incapable de dialoguer. $\mathrm{Si}$, face à une telle situation, il fuit, laissant derrière lui une femme en manque et en stress. D'où vient donc la violence dont elle est victime? Pourquoi la société tolère ce genre de harcèlement moral? Pourquoi c'est toujours la femme qui endosse la responsabilité de la mésentente? Pourquoi la société la considère comme origine des troubles?

A travers cette étude, nous avons tenté de découvrir deux réponses valables à cette problématique. L'une est scientifique, la seconde est sociale.

\section{d) Harcèlement moral contre la femme:}

Sa peur des représailles virtuelles d'un mari disposé par nature à la violence, rend l'épouse prisonnière de sa situation de mère. Elle veut préserver la stabilité du couple et des enfants, alors, elle se tait. Mais plus elle se tait, et fait rentrer sa colère, plus, elle encourage le mari à augmenter la dose de violence envers elle et donne lieu à le harcèlement moral:

" Devant un conflit relationnel et un rejet, le cerveau féminin s'alarme plus que le cerveau masculin (......). chez les femmes, le conflit risque de déclencher une cascade de réactions chimiques qui vont les chambouler et susciter de la peur et des tensions. La simple perspective d'un conflit se traduit dans le cerveau par une menace sur la relation et s'accompagne d'une inquiétude à l'idée que la prochaine conversation (...) puisse être la dernière. " ( brizendine, 2008, p. 136)

Quand la femme opté pour le mutisme, le mari, de par sa nature, prend la relève dans l'escalade de la tension. Pus elle se tait, plus elle encourage le mari à augmenter la dose de violence et d'ignorance envers elle. Autrement dit, elle ne s'explique pas pour se faire comprendre ou plutôt, elle n'arrive pas elle aussi à s'exprimer 
clairement ce qui la fait perdre de terrain. Elle lui offre l'occasion de calomnier, de frapper, de menacer ou de fuir vers une autre relation. Invectiver et menacer viennent se ranger sous la rubrique de le harcèlement moral. Frapper est une violence physique. Fuir pour retrouver une autre relation est une violence indirecte contre la femme. Abandonner le couple comme un châtiment pour l'épouse est une violence sexuelle indirecte. Après tout cela, on considère la femme comme la première responsable de l'écroulement du couple???

La société garde sous silence la violence innée de l'homme. Elle ignore également ses fautes. Elle le rend toujours gagnant de toutes les situations de conflit quelle qu'elle soit. La société ignore totalement la part de responsabilité de l'homme. Elle passe sous silence qu'il est plus violent que la femme, la société ignore également que la disposition naturelle des hommes à la violence déclenche chez eux une vive réaction d'agression physique. Ces réactions peuvent aller du harcèlement moral jusqu'à la violence criarde et affichée.

\section{e) La neurobiologie ${ }^{5}$ prouve la violence masculine:}

La neurobiologie nous a fourni des preuves sur la disposition naturelle des hommes à la violence. Dr. Brizendine a expliqué que le cerveau masculin, dès l'adolescence, est dominé par l'hormone de la testostérone qui le pousse à être plus agressif que le cerveau féminin:

" Hommes et femmes produisent de la testostérone, mais chez l'homme, la production est dix fois plus importante." ( brizendine, 2008, p. 62)

Elle ajoute que: "les hommes sont en moyenne 20 fois plus agressifs, et il suffit de jeter un oil dans une prison pour s'en convaincre. "(brizendine, 2008, p. 62)

Les preuves sont probantes que le cerveau masculin est naturellement branché sur la violence. Les deux sexes possèdent l'hormone de l'agressivité, cependant la différence de quantité entre les deux est exorbitante. 
"les hommes en possèdent de 10 à 100 fois plus que les femmes." ( brizendine, 2008, p.p. 158-159)

De ce qui précède, nous pouvons conclure que, tant que les cellules cérébrales sont envahis par cet hormone, tant que l'homme demeurera plus enclin à l'agression. Et tant que la société ignore et laisse passer cette agressivité surtout lorsqu'elle est pointée contre la femme, tant que l'agressivité durera et tant que cette problématique durera. Cette agressivité peut s'afficher sous forme de paroles blessantes, d'actes de manque de respect, de coups de poings, coup de pieds.

\section{III) TROISIÈME point: Origine sociale de la violence:}

\section{a) installation de l'hégémonie par la violence:}

Michael Kauffman avait précisé 7 raisons sociales claires pour expliquer pourquoi la femme est toujours cible à l'oppression depuis la nuit des temps jusqu'aux sociétés les plus modernes. Kauffman explique que la violence masculine est née depuis les sociétés patriarcales où la suprématie sur les autres membres du groupe ne prenne place que par la violence. Pour fonder la suprématie des uns sur les autres (entre hommes tout d'abord), cette phase doit passer par le combat de l'autre et la marque d'un but. Les hommes entre eux apprennent à se servir de la violence. L'hégémonie s'installe au moyen de la violence pour désigner le plus fort: " C'est la manière dont elle a été intégrée dans nos idéologies et nos structures sociales" ( kauffman, 1985, p. 1)

Ainsi, l'environnement favorise t'-il ce genre de comportement violent qui, petit à petit, prend racine dans la société comme ayant valeur de facto. En société, la violence masculine requiert la place d'une idéologie. Pour être fort, il faut être violent. Un concept qui prend racine et s'ancre dans l'idéologie masculine dans sa manière de vivre en société: "Il en résulte notamment que les hommes (internalisent) la violence". (kauffman, 1985, p. 2) 
socialement parlant, la violence est aussi vénérée dans la logique masculine par les différents genres de sport: boxe, karaté, thaikondou, konghfou, extra...

La violence est ainsi, non seulement valorisée mais aussi glorifiée et récompensée: "Généralement, les hommes ne détestent pas les conflits interpersonnels et les compétitions; cela peut même les stimuler." ( kauffman, 1985, p. 2)

Ayant testés que la violence envers autrui réalise des bénéfices considérables à celui qui s'en sert: devenir chef d'équipe, chef de bande, chef de famille, l'homme cherche à perpétuer le privilège:

"Dans un tel contexte, la violence réelle ou sous forme de menace devient un moyen de perpétuer la moisson de privilèges et l'exercice du pouvoir. C'est à la fois un résultat et un moyen d'atteindre un objectif." (kauffman, 1985, p. 2)

Ayant franchi un premier pas, la violence que commet un homme envers une femme traduit son désir de perpétuer ce genre de privilège déjà acquis. Elle demeure l'expression du droit à être toujours servi par sa femme. Kauffman analyse minutieusement ce fait en rapportant:

" (...) ce n'est pas la répartition inégale du pouvoir qui conduit à la violence mais bien un sentiment conscient ou inconscient de privilèges dûs." ( kauffman, 1985, p. 2)

\section{b) Test de virilité:}

Par crainte de ne pas détenir le pouvoir, de ne pas perpétuer les privilèges, les hommes ont paradoxalement contribué à construire leur propre prison. Pour garder la main haute, cela implique une force interne pour être à même d'une société qui exige cette supériorité de l'homme. Celui-ci se trouve dans l'obligation de se blinder en vue d'inspirer la crainte vis à vis de l'autre. Ce genre d'obligation psychique affronte l'homme à une peur interne et nondit, expression d'une insécurité personnelle à ne pas réussir le test de " virilité" devant la société. La peur d'un tel échec active les 
ressources de l'agression surtout vis à vis de la partenaire conjugale. Comme si, quand il n'use pas de violence, cela amoindrit sa masculinité. Les hommes se trouvent confrontés à une "spirale de crainte." ( kauffman, 1985, p. 2) Ils ne trouvent d'issue que dans l'agression. La violence joue donc ici le rôle d'une compensation psychique. Kauffman explique cette idée en avançant:

"c'est une façon de rétablir l'équilibre masculin, d'asséner à soimême et aux autres les preuves de sa masculinité. 2017 ( kauffman, $1985, p .4)$

En conclusion, la société qui permet que le mari bat sa femme est une société malade mais les jeunes garçons éduqués dans un tel milieu grandissent en trouvant normal ce genre de comportement. Bref, perpétuer et internaliser la violence comme norme comportementale s'avère donc une question de survie pour sauver la face.

\section{c) D'une société malade à un couple malade:}

Cette société malade ne peut engendrer que des relations conflictuelles. Nous étions énormément étonnée lorsqu'aucun des deux partenaires n'a opté pour la séparation dans le roman de Ben Jelloun. Le romancier a décrit une atmosphère conjugale irrespirable. Leur haine respective était affichée jusqu'à un degré qui révolte le lecteur. Alors, une question s'impose : pourquoi supporter une vie aussi envenimée que cela?

" Le peintre et sa femme vivaient un véritable enfer. La maison était leur champ de bataille, leurs amis étaient pris à témoin, leurs familles des arbitres fort peu impartiaux".( Jelloun, 2012, p. 346)

Pourquoi personne n'a envisagé la séparation? Quant à la femme, elle était une machine à engendrer une haine criarde.

"je le regarde et je vois en lui avant tout le traître et le monstre qui a exploité mes jeunes années et puis m'a abandonnée. " ( Jelloun, 2012, p. 346)

A maintes reprises, elle a voulu le quitter, cependant elle n'a jamais eu le courage de rompre. Au contraire, elle s'accroche de plus 
en plus dans un engrenage infernal de possession. Elle a subi une violence sexuelle feutrée lorsque son mari n'a pas voulu d'elle et lorsqu'il l'a trahi à plusieurs reprises. Elle était également victime de la violence sociale qui laisse passer sous silence cette infidélité de la part du mari et la sanctionne vis à vis de l'épouse. Elle était encore une fois emprisonnée dans les barbelés de la tradition sociale qui lui interdit de trahir son mari pour se venger. Ce fut un engrenage de violence implicite consenti par une société masculine oppressive.

En ce qui concerne le mari, le peintre était à bout de force. Chaque fois qu'il réalise qu'il doit quitter cette vie infernale, il ne s'exécute pas. Il ne trouve en lui nullement le courage de quitter. Pour quelle raison s'accroche t'- il à cette vie maladive?

"N'en pouvant plus, perdant tout contrôle, le peintre avait jeté un verre d'eau au visage de sa femme. Elle avait immédiatement répliqué en lui lançant son verre de vin. Ses yeux ne voyaient plus; il s'était retrouvé dans le noir durant quelques secondes. Tout le restaurant avait suivi la scène. " (Jelloun, 2012, p. 346)

A maintes reprises, il aboutit à l'impossibilité de la vie commune; il se disait à lui-même:

"Quand la violence s' installe dans un couple, (...) la vie en commun n'est plus possible; tout le reste n'est que rafistolage et mensonge à soi. Divorcer alors est la seule solution. " ( Jelloun, 2012, p. 346)

Il paraît que le peintre était psychiquement dépendant lui aussi. Un certain lien incompréhensible résistait à la prise de ce pas final:

"Il comprit que l'important était de sauver sa peau, de prendre la fuite et d'en finir avec ce couple où il n'avait plus sa place ni son rang." ( Jelloun, 2012, p. 114)

Il se rend enfin compte qu'ils se sont trompés tous les deux. Il reconnaît qu'il aurait dû se méfier davantage de ses sentiments. Il comprend enfin que la différence entre leurs deux mondes était 
immense. Il reconnaît également qu'ils étaient venus de deux planètes différentes:

" Je suis l'unique responsable de cette défaite. Notre différence était plus profonde et plus grave: tout au long de notre vie commune, nous n'avons pas vécu la même histoire et jamais nous ne l'avons reconnu." (Jelloun, 2012, p. 114)

Aboutissant de la sorte à un chemin sans issu, la solution logique aurait été de se quitter, mais la dépendance au sein de ce couple avait soulevé des interrogations. Nous nous sommes penchée sur ce genre d'addiction pour pouvoir comprendre qu'est ce qui peut pousser un couple qui se hait à consentir à vivre une vie malheureuse sans avoir le courage de mettre terme à cet engrenage de souffrance.

\section{IV- QUATRIÈME point:}

\section{Carences psychiques des époux:}

\section{a) signes de carence affective chez l'épouse:}

Hélène Roubeix nous explique que la carence affective dans l'enfance peut être à l'origine de cette dépendance illogique. De part et d'autre, les deux conjoints ont donné des signes de carences affectives ou de manque de consistance psychique : "Parfois les carences affectives sont si grandes qu'elles nous amènent inévitablement à vivre des relations amoureuses toxiques et destructrices." ( roubeix, 2008, p. 2)

Il paraît que les deux conjoints souffrent peut-être d'une fragilité psychique, une certaine inconsistance dans la construction de leurs personnalités réciproques:

" L'enfant perdu et mal aimé veut à tout prix retrouver son père et être aimé par lui comme il aurait été juste qu'il le soit autrefois. (....) Il ne voit pas que cet homme ressemble étrangement à son père, non seulement physiquement mais psychologiquement: la même froideur, la même rigidité, la même incapacité à être proche affectivement et à créer des liens. Après avoir cru être comblé, l'enfant intérieur ne peut supporter le manque. "( roubeix, 2008, p. 3) 
Souffrant irrémédiablement de ce manque affectif, la femme du peintre, sous l'illusion d'un coup de foudre, accepte le mariage avec un homme de quatorze ans de plus qu'elle et encore il appartient à un monde totalement paradoxal: "Les souvenirs des insupportables humiliations dont elle avait été victime dans son enfance parce qu'elle était de condition modeste lui revenaient, comme une blessure secrète qui se rouvrait d'un coup. " (jelloun, 2012, p. 61)

Il nous semble que cette carence affective et cette fragilité interne ont poussé cette dame à supporter l'humiliation. Elle est tombée, de son propre gré, dans les barbelés d'un mari narcissique qui a voulu la façonner à son gré. Tel est le cas d'une femme qui n'a pas trouvé l'amour au sein de sa famille à l'enfance, et elle a nourri une illusion de le retrouver ailleurs. Elle est tombée proie à un homme pervers. Elle a accepté de se placer en position de victime:

" Je suis comme ça; mon père a cesse de m'embrasser à 3 ou 4 ans". (jelloun, 2012, p. 61). Cela prouve qu'elle souffrait vraiment d'une carence affective .

\section{b) PERVERSION narcissique du mari.}

Dans son colloque "les sens du mal" tenu à Bruxelles en 2010, Dr. M. Cautaerts explique que "la perversion narcissique est "cette peste, (qui) exerce ses ravages et ses saccages en tous lieux" ( cautaerts, 2010, p. 7)

Ce sont des manipulations et des harcèlements qui ont été nommés, à juste titre, par Marie France Hirigoyen: Meurtres silencieux. Le pervers narcissique,comme l'indique sa qualification, souffre d'une "inflation psychique." il possède un "ego surdimensionné. " ( cautaerts, 2010, p. 7)

Le pervers souffre des troubles d'identité et d'estime de soi ${ }^{6}$. Il souffre des troubles de l'identité. L'expression "Pervers narcissique" est utilisé en psychopathologie pour désigner les individus atteints d'un narcissisme exacerbé et des traits de perversion morale. La 
perversion narcissique est un mécanisme de défense où l'homme atteint essaye de préserver son identité fragile en blessant l'autre. Dr. Cautaerts ajoute que: "le pervers narcissique se préserve d'une blessure de l'identité." (cautaerts, 2010, p. 19)

Il utilise l'autre comme un objet ou un ustensile où il déverse ses manies:

"l'autre est une chose, un ustensile". " pour le pervers, l'autre n'a pas droit au statut de sujet. Il n'est qu'un ustensile." (Cautaerts, 2010, p. 20)

La personnalité du pervers dénie toute existence de l'autre comme une entité propre. Elle n'accepte jamais cette existence. Cet autre n'existe qu'en fonction de ses désirs. Il est là seulement pour servir son égoïsme. La femme du peintre avance que celui-ci la considérait comme un bagage duquel il préfère se soustraire:

"(...) Il s'est permis de dire qu'il ne voyageait jamais avec moi parce qu'il aimait se déplacer sans bagages, librement, sans être encombré d'une femme qui lui poserait, inévitablement, mille problèmes. "(Jelloun, 2012, p. 303)

Le pervers, pour se défendre, attaque sa victime pour démolir son identité et pouvoir par la suite, la traîner dans son engrenage: " (il) jouit de faire souffrir l'autre." ( cautaerts, 2010, p. 6)

En tant que bourgeois, il a bien choisi sa victime: jeune, de caste inférieur, une famille analphabète et psychiquement fragile. Une proie idéale pour avoir le plein pouvoir sur elle. Peut-être, inconsciemment, il l'a choisie beaucoup plus jeune avec une différence de 14 ans pour la traiter comme un jouet, pour la sculpter à sa façon:

" Il pensait avoir épousé une petite bergère qui ne dit mot, qui baisse les yeux et avale des couleuvres" ( Jelloun, 2012, p. 303)

Dr. M. Cautaerts nous explique que le pervers, derrière son apparente toute puissance, il reste fragile "car il a sans cesse besoin de victimes à humilier, voire à détruire, afin d'affirmer sa propre grandeur. " (cautaerts, 2010, p. 6) 
De ce qui précède, il paraît clair que le peintre avait besoin de se sentir en sécurité quand il a choisi la jeunesse. Il avait besoin de se sentir grand et pour la manier à son gré, il devait user de beaucoup de charme et de séduction. Cette séduction n'était que le filet du prédateur pendant la période des fiançailles:

" la séduction consiste à tirer quelqu'un hors de son chemin. Le pervers narcissique séduit sa future victime afin d'attirer sur lui la projection de son idéal du moi." ( cautaerts, 2010, p. 21)

Et comme son besoin de glorification a besoin d'être soutenu, le pervers n'a d'autre solution que d'ôter à son partenaire ( ici, sa femme) toute tentative d'être soi. Il doit effacer son identité pour la faire tourner dans l'engrenage de son narcissisme.

Dr. Cautaerts présente le tableau suivant pour expliquer la relation du conscient et de l'inconscient du pervers face à sa victime et vice versa.

Relation du CONSCIENT du PERVERS au CONSCIENT DE LA VICTIME:

PERVERS NARCISSIQUE $================>>$ VICTIME

Relation d'amour à condition d'être prouvée $<<<==============$

Croyance en une relation normale réciproque de couple

Relation de L'INCONSCIENT DU PERVERS A L'INCONSCIENT DE SA VICTIME:

PERVERS NARCISSIQUE VICTIME

Emprise 


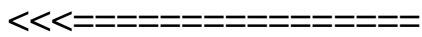

\section{se fait médium malléable vu la demande transferielle du pervers qui active le maternel chez sa victime.}

Quand le pervers active les sentiments maternels de son épouse ou bien de sa victime, il sollicité son attention et sa couverture de protection. Il essaye par tous les moyens protéger son Moi narcissique vulnérable. Alors, il use du charme pour séduire, pour mieux engluer la victime dans son sillage. Dès que la victime ose se soustraire à cette emprise, éclate, alors, la colère du pervers.

Cet éclatement a eu lieu dans la vie du couplé, objet de notre étude, lorsque la femme du peintre a osé dire: NON.

Lorsqu'elle a osé hausser la voix pour dire NON à la soumission, à la manipulation, à la transformation en une esclave. Elle a dit NON à l'effacement de son identité propre. Son mari qui a voulu faire d'elle un jouet en effaçant ses goûts, ses désirs et ses besoins, éclate de colère. Il a voulu effacer sa propre existence pour ne vivre qu'à travers lui:

D'ici commence le harcèlement du mari envers elle. Il savait comment provoquer chez sa femme des tempêtes émotionnelles successives: "Il avait une façon vicieuse de provoquer en moi ce qu'il y a de pire, ce sentiment affreux mais légitime qui rend les couples fous. Sa perversité se manifestait de manière sournoise, évidemment. " ( Jelloun, 2012, p. 319)

Un peu plus loin, elle ajoute:

"Foulane avait l'art de savoir m'atteindre dans mon orgueil, il allait chercher les blessures enfouies au fond de mon enfance et $y$ retournait le couteau pour me faire mal, très mal; (...)." ( Jelloun, 2012, p. 320)

Il avait cet art de provoquer en elle un sens aigu de jalousie, un sentiment non seulement contrariante mais aussi, un degré extrême de harcèlement moral: "Voilà, ma jalousie n'était rien d'autre que de la colère, de la contrariété poussée à l'extrême. " ( Jelloun, 2012, p. 322) 
Psychiquement malade, le pervers se plaît à prendre la position d'une victime. Après l'attaque et la provocation, il se donne le droit de se plaindre comme s'il était lui-même la victime. Comme il jette toute la responsabilité de tout ce qui ne marche pas dans sa vie de couple sur sa femme, il prétend qu'à son absence, tout le monde se repose de la contrariété qu'elle provoque.

Nous pensons que la faiblesse de ce couple était donc due aux carences affectives chez l'épouse qui a accepté inconsciemment de se placer en position de victime. D'autre part, la déviation psychique chez le mari a achevé l'échec du couple. Si cette épouse avait besoin d'être sécurisée dans les bras d'un mari normal, elle aurait réussi sa vie conjugale. Mais le fait qu'elle soit tombée dans les barbelés d'un pervers, a compliqué leur vie à l'extrême. Le grand problème d'un pervers, c'est qu'il considère que la présence de la femme dans sa vie est une atteinte à son intégrité propre. Il vit la relation conjugale sous le signe de la MENACE DE SON INTEGRITE. MARIE FRANCE HIRIGOYEN avait déjà expliqué ce fait dans son œuvre " le harcèlement moral, la violence perverse au quotidien"7 en disant que:

"La présence de l'autre est vécue comme une menace, pas comme une complémentarité" ( hirigoyen, 2003, p. 20)

Le pervers essaye toujours de dévaloriser sa victime pour se sécuriser intérieurement. Cette disqualification est nécessaire pour que le partenaire (ici l'épouse) se révèle en deçà de ses compétences, incapable de répondre à toutes les exigences du pervers "or le pervers ne peut jamais se déclarer satisfait car alors la victime aurait une Valeur!" ( hirigoyen, 2003, p. 21) Ce qui est inacceptable pour le pervers car son jeu deviendra un "non-jeu".

De la sorte, le cercle infernal s'est raffermi sur la position psychique détériorée des deux époux. L'une souffrant de carence affective cherche à se sécuriser dans un mariage où toutes les données sonnent le glas de l'échec. L'autre, psychiquement malade, avait besoin de dévaloriser l'autre pour exister. 
Ben Jelloun, avait certes des messages multiples à transmettre. Des messages, parfois implicites, parfois explicites. A travers ce couple infernal, il a voulu montrer au lecteur comment la vie peut s'avérer irrespirable sans communication saine et constructive. En critiquant les critères sur lesquels les dames fondent le mariage, il dit: " Aucune ne parla de jardin secret, de dialogue, de construction quotidienne du couple dans le respect des différences, sans exclure pour autant les dissensions" ( Jelloun, 2012, p. 175)

Pour défendre la cause de la femme, il a voulu peut- être lancer un clin d'œil aux hommes en critiquant sa société marocaine:

" Les femmes, c'est par la douceur qu'il faut les garder, pas par les coups de poing. C'est ce qui fait que chez nous, au Maroc, nous sommes en retard sur bien des sociétés. " ( Jelloun, 2012, p. 175)

Il se fait porte-parole de toute femme humiliée et dévalorisée en lançant vers la fin de ce roman noir:

" Nous ne sommes pas des objets de leur désir. (...) nous ne serons plus soumises à l'homme qui se croit fort, nous ne serons plus humiliée par ses prétentions, par ses ambitions qui nous sacrifient et nous piétinent" ( Jelloun, 2012, p. 338)

Il lance également une menace à la figure des hommes et de la société masculine qui ne cesse de harceler moralement la femme sous diverses formes: " Marchons sans nous retourner, car ces hommes qui nous exploitent savent que maintenant nous sommes plus fort qu'eux, décidées à prendre notre destin, notre vie et nos énergies en main." ( Jelloun, 2012, p. 339)

\section{Conclusion:}

Nous voici, avons abouti à l'étape finale de notre recherche pour en mettre en exergue les résultats.

Nous pensons que la problématique de l'incompréhension entre l'homme et la femme demeurera présente jusqu'au jour du jugement. Ce que nous avons essayé d'expliquer n'était qu'une simple tentative d'éclaircissement. Les processus mis en place par les sociétés patriarcales ne seront jamais effacés dans leur totalité surtout qu'ils 
ont fonctionné avec une réussite éclatante pendant des siècles. Placer la femme dans l'engrenage de la servitude a duré pendant des siècles au profit de l'homme avant que la gent féminine ne sache l'instruction. Ils ont abusé de son ignorance, de son illettrisme, de son émotivité. Ils ont tourné des couteaux envenimes dans ses plaies sans mercis. Ils ont profité d'elle au maximum, des profits domestiques tout d'abord, des profits sexuels et financiers par la suite. Lorsque la femme s'est libérée de l'emprise masculin par le travail, ils ont profité d'elle financièrement. Par tous les moyens, ils l'ont agressée pour perpétuer leurs privilèges. Même après l'enseignement, l'instruction, la libération sociale de la femme, les gènes masculins ont refusé intérieurement que celle-ci soit libérée de leurs jougs. Finalement, la science moderne a pu nous éclaircir sur des réalités cachées. Nous avons découvert une très grande différence entre le fonctionnement du cerveau féminin et du cerveau masculin. Le premier est sentimental, plus disposé à l'empathie et à la communication, le second est plus disposé à l'agressivité. Le maintien de l'incommunicabilité provient de cette différence importante mais mal connue des deux sexes. La maladie psychique peut facilement toucher les deux sexes, mais le plus agressif use de plus de violence pour sortir gagnant. Nous avons aussi découvert le processus de la perversion morale. Ce processus de déviation psychique qui pousse l'homme à jeter la responsabilité de tout échec marital sur le compte de la femme. Un mécanisme de harcèlement noir qui attaque la victime comme une pieuvre pour la déstabiliser et lui arracher toute confiance en elle-même. Un meurtre psychique à sang froid qui atteint la femme dans sa dignité et son assurance en vue de la traîner davantage dans le sillage de sa dominance. Toute tentative de perpétuer les privilèges de l'homme sur la femme était soutenue par une société permissible, une société qui valorise et récompense la violence et la suprématie de l'agression sur le dialogue. Une société qui ne sait pas être juste. Les hommes maintiennent leurs privilèges grâce à leur disposition naturelle à l'agressivité; les femmes n'arrivent pas à arracher la justice de cette société sexiste à cause de leur faiblesse émotionnelle. La mère de famille préfère garder le silence devant le harcèlement moral d'un mari plus ou moins atteint de perversité, soit morale, soit psychique, pour maintenir l'équilibre de sa famille. L'épouse, de peur de perdre 
son équilibre psychique, reste muette devant le harcèlement. Toujours la société profite de la vulnérabilité émotionnelle de la femme pour donner raison à l'homme. La solution d'une telle problématique est extrêmement difficile. Dorénavant, la femme doit travailler sur l'information, sur la propagation de cette différence scientifique entre elle et l'homme. Elle doit également ne laisser passer sous silence en aucun cas sa transformation en une victime. La science doit être vulgariser pour lutter contre la victimisation de la femme. La femme ne doit pas se laisser faire, elle ne doit pas également se taire. Dorénavant, la responsabilité incombe à toute mère qui doit implanter une confiance intrinsèque dans l'armature psychique et mentale de sa fille pour la rendre robuste émotionnellement. Quand cette force aura lieu, la jeune fille ne permettra à personne de la transformer en victime à travers des mécanismes de perversion noire.

Ben Jelloun s'est fait le porte-parole de la femme atteinte de violence dans une société permissive pour porter un cri contre l'injustice. Son roman est une révolte en soi. Un roman noir de haine a séduit le public par un titre illusoire. Tout le sarcasme du romancier était condensé dans le choix de ce titre. Il a présenté à la société un tableau et un cadre de vie ordinaire: un couple comme des milliers d'autres fonde un espoir de vie heureuse sans avoir édifier une base solide pour le faire réussir. Nous pensons que Ben Jelloun, a voulu lancer un clin d'œil rapide à une société qui se démantèle sans s'en rendre compte. Il a voulu attirer l'attention que cette désintégration de la société a besoin de solutions, de compréhension, de communication et peut-être aussi d'abnégation de part et d'autre pour que le couple puisse passer sur les difficultés journaliers qui s'entassent pour construire le mur d'incompréhension entre les époux. Il a ausculté des maladies psychiques inconnus de la société: la perversion psychique, narcissique et sexuelle des maris, l'ingérence des familles dans la vie du couplé, les assises fragiles sur lesquelles on choisit le partenaire de la vie. Il a voulu mettre l'accent aussi sur le choix que chaque fille pauvre fait pour un mari riche pour illustrer que l'entente est mieux que l'argent. Il a voulu également critiquer la femme dont l'émotivité est accentuée pour lui rappeler que la gent féminine possède le génie de la patience. 
Bref, Ben Jelloun s'est révélé à nos yeux comme romancier intéressé à fond par les problèmes de la société et surtout ceux ayant trait à la balance de la justice qui pèse les actes et leurs conséquences dans les relations entre les différents types de personnes.

Nous avons découvert le harcèlement moral et psychique qui nuit fatalement aux couples. En lisant à tête reposée l'œuvre de MarieFrance Hirigoyen, nous avons découvert que les pervers narcissiques ou pervers sexuels peuvent être traiter d'une certaine façon pour faire stopper ou amoindrir les dégâts de leur harcèlement. Et comme ce traitement dépasse les limites de ce modeste travail, nous avons seulement voulu tirer au clair que toute problématique peut avoir des solutions grâce à la nouvelle science.

Les résultats de la présente recherche nous ont étonnée personnellement parce qu'ils nous ont ouvert la porte à la neuroscience, un domaine de recherche ultra fascinant. En ayant accès à l'imagerie cérébrale des personnes atteintes de maladies psychiques s'ouvre devant nous un monde qui a été totalement clos, un horizon incontrôlable d'information scientifique qui éclaire l'avenir grâce à l'évolution de la science. La compréhension de la formation génétique du cerveau masculin et féminin nous a beaucoup aidée à mieux analyser cette relation problématique. La science éclaire le chemin vers plus d'entente. Espérons qu'elle nous fait découvrir dans le futur des moyens pratiques en vue de minimiser les divergences entre les deux sexes.

\section{$\underline{\text { Abstract: }}$}

Nous nous intéressons à analyser les raisons sociales et psychologiques qui maintiennent la violence conjugale contre la femme au sein du couple en particulier et au sein de la société en général. Qu'est ce qui fait de la femme un objet permanent d'attaque de la part des hommes? Nous cherchons dans les origines sociales de cette problématique irritante ainsi que dans les explications scientifiques modernes qui peuvent nous fournir une réponse valable à ce dilemme. La psychanalyse, la socfascinants la neurobiologie nous ont fournies des résultats fascinants. 
${ }^{1} \mathrm{Cf}$. Roubeix, De la dépendance amoureuse à la liberté d'aimer, Op.cit.p.37

${ }^{2}$ Cf., Hélène Roubeix, op.cit.

${ }^{3}$ Brizendine, les secrets du cerveau féminin, Paris, Grass et, 2008. Traduit de l'américain par Marie-France Girod.

${ }^{4}$ Kaufman (Michael), the construction of Masculinity, Toronto, Oxford University Press, 1985.

${ }^{5}$ La neurobiologie est une science qui cherche la compréhension biologique, moléculaire et cellulaire des troubles mentaux. "Le mot est né en 1970 et désigne l'ensemble des disciplines scientifiques et médicales étudiant le système nerveux et incluant la psychiatrie et la psychologie clinique. Il intègre une double approche d'une part psychobiologique qui recherche les bases biologiques des comportements et d'autre part psychologique qui étudie le fonctionnement du psychisme" Roubeix, op.cit.p.180

${ }^{6}$ nous attirons ici l'attention du lecteur sur la nature des personnages présentés par Ben Jelloun qui n'a cessé de dessiner des images de personnages masculins souffrant d'une perte de l'identité comme reflet de la non consistance psychique des jeunes émigrés.

${ }^{7}$ Hirigoyen ( Marie-France), le harcèlement moral, la violence perverse au quotidien, Paris, la découverte, 2003. 


\section{Bibliographie:}

1) Le corpus: Le bonheur conjugal, Paris,Gallimard, 2012.

2) Ouvrages spécialisés en neuroscience:

Bourassa(Michelle), Le cerveau nomade,canada,presses univ. d'Ottawa.

Brizendine (Louann), Les secrets du cerveau féminin, traduit de l'américain par Marie France Girod, Paris, Grasset, 2006.

Kolb (B), Wishaw, cerveau et comportement, Paris, De Boeck, 2002.

\section{3) Ouvrages spécialisés en psychologie:}

Gazzanga, ivry, mangue, neurosciences cognitives, la biologie de l'esprit, Paris, de boeck université, 2001.

Hirgoyen ( Marie France), Le harcèlement moral ou la violence perverse au quotidien ,Paris, la Découverte, 2003.

House (olivier), Mazoyer (Bernard), tzourio (Natalie), cerveau et psychologie, Paris, puf, 2003.

Reynaud(Michel),l'amour est une drogue douce en général,Paris,Laffont, 2005.

Roubeix ( Hélène ), De la dépendance amoureuse à la liberté d'aimer, Paris, Saint Germain, Eyrolles, 2008.

\section{4) ESSAIS EN SÉMIOTIQUE:}

Fontanille ( Jacques ), "pratique et éthique: la théorie du lien, in Prothèse, 2008. Volume 36 no. 2 , université de Limoges, institut universitaire de France.

\section{5) ESSAIS EN SOCIOCRITIQUE:}

Angenot ( Marc), "Que peut la littérature?" Sociocritique littéraire et critique du discours social paru dans l'ouvrage collectif " la politique du texte, enjeux socio critique pour Claude Duchet, presses universitaires de Lilles 1992.

Michael Kaufman, "The Construction of Masculinity and the Triad of Men's Violence," in M. Kvil éd. Beyond Patriarchy: Essays by Men on Pleasure, Power and Change, Toronto: Oxford University Press, 1985. Réimprimé en anglais par Laura L. O'Toole et Jessica R. Schiffman, Gender Violence (New York: NY University Press, 1997) et cité par Michael S. Kimmel et Michael A. Messner, Men's Lives (New York: Macmillan, 1997) ; en allemand dans la collection BauSteineMänner, 
Kritische Männerforschung (Berlin: Arument Verlag, 1996) ; et en espagnol dans l'ouvrage Hombres: Poder, Placer, y Cambio (Santo Domingo: CIPAF, 1989). (3) Michael Kaufman, Cracking the Armour: Power, Pain and the Lives of Men Popovic ( Pierre ), " la SOCIOCRITIQUE, définition, histoire, concepts, voies d'avenir" , in Pratiques no. 151- 152, décembre 2011.

6) Articles ayant trait à la psychologie:

Bahi ( Mohamed), " la ville e ( s ) t la femme: deux corps agressés", in

Femmes et villes, textes réunis et présentés par Sylvette Denefle, collection perspectives. Presses Universitaires François-Rabelais, Maison des Sciences de l'Homme «Villes et Territoires»

Barthes (Roland), "théorie du texte", in Le plaisir du texte, Paris, 1973, p. p. 1 - 11 .

Collot ( Michel ), "la textanalyse de Jean Bellemin- Noël" , in Littérature, no $=58,1985$, le savoir de l'écrit , p. p. 75- 90 .

Gérard ( Jessica ), Pourtois ( Véronique ), cours de psychologie, AMEPS U.F. conceptuelle 1, 2010- 2011, Erquelinnes.

Lorenz ( Suzanne), " favoriser le changement chez des auteurs de violence dans le couple: le rôle du travail de groupe", à periodical of FESET/ revue de FESET - journal européen de l'éducation sociale, p. 73- 89.

Mokhtar ( Hafida Ait), " le personnage féminin: glorification ou anonymat dans la nuit sacrée de Taher Ben Jelloun", Synergies, Algérie, no. 1, 2010, p. p. 195- 201

Rouge (Dominique), les lectures psychanalytiques des oeuvres littéraires, université pédagogique de Cracove, Pologne, no. 8, 2011, p. p. 13 - 20.

\section{7) Thèses et Mémoires consacrés à l'oeuvre de Ben Jelloun:}

Aldahham ( Naser), Marcel Pagnol et Taher ben Jelloun: enjeux culturels de l'apprentissage de la masculinité, université de Concordia, Montréal, Québec, Canada, 2012.

Berclaz ( Michel), Agressivité, Hostilité et Violence, Genève, HUG, 2001.

Carlsward ( Linda ), la quête de l'identité dans l'enfant de sable de Taher ben Jelloun, Franska D - uppsats, 2007, Karlstads , universitet.

Kauranen ( Tytti ), les métaphores dans deux oeuvres de Taher Ben Jelloun, mémoire de maîtrise en philologie romane, université de Jyvaskyla, 2001.

Qadeesh ( Bassima ), l'identité féminine dans L'enfant de Sable et la nuit sacrée, University of Georgia, Athens, 2006. 
Samokhina ( Daria), le phénomène de l' Hibridite et du Mimétisme dans des espaces narratifs du Maghreb: une identité, est- elle possible?, Notre Dame, Indiana, 2005.

\section{8) COLLOQUE EN PSYCHANALYSE:}

Cautaerts ( Dr. M), Mortelles blessures d'amour, doubles narcississiques, harcèlement et perversions, présenté au colloque Les sens du mal à Bruxelles le 4 décembre 2010.

\section{9) Rapport et enquête:}

Rapport de l' Organisation Mondiale de la Santé sur la violence, "Violence et Santé", rapport de l'enquête réalisée par l'institut BVA à la demande du Haut Comité de la Santé publique en République Française.

Maryse Jaspard et l'équipe Enveff, Nommer et compter les violences envers les femmes : une première enquête nationale en France , *volume 346, 2001.

Michael popp, Ph.D.Judiple, La violence familiale chez les autochtones au Canada. ÉTUDE rédigée pour le compte de la Fondation autochtone de guérison.

10) Cours de psychologie:

Belanger ( Jean ), psychologie de la personnalité, université du Québec, Montréal, département de psychologie, 2004.

Roy ( Vincent), Master 1 et 2 , apprentissage, acquisition et construction des connaissances, université de Rouen, 2014.

\section{1) Webiography:}

http://www.violencesfaitesauxfemmes.com/ewb_pages/p/presentationgrande-cause2010.php. La loi du 9 juillet 2010 relative aux violences faites spécifiquement.

http:// psychobiologierouen.free.fr.

http://lecerveau.mcqill.ca /

WWW. taharbenjelloun. Org.

\section{Table de matière :}

\section{Introduction}

Choix du sujet, problématique, Approches, Plan de travail.

Premier point: étapes de la constitution du couple:

I) Première phase du mariage. a) fusion de l'amour . b) résurgence des malentendus. 
II ) Deuxième phase du mariage:

conflit et contre dépendance

Différence de culture

Manque de respect

Manque de confiance

Deuxième point: différence anatomique et neurologique entre le cerveau masculin et féminin:

a) disposition naturelle à la communication du cerveau féminin.

b) armature mentale rigide du cerveau masculin .

c) la violence feutrée contre la femme.

d) le harcèlement moral de la femme.

e) la violence masculine.

Troisième point: les raisons sociales de la violence.

a) installation de l'hégémonie par la violence.

b) test de virilité.

c) d'une société malade à un couple malade.

Quatrième point: carences psychiques des deux époux.

a) signe de carence affective chez l' épouse.

b) perversion narcissique du mari

Conclusion

Bibliographie

Table de matières. 